\title{
Acompañamiento Pedagógico Digital para Docentes
}

\section{Digital Pedagogical Accompaniment for Teachers}

Marcela Zeballos ${ }^{1}$

\section{ఠ} EDICIÓN: 进-CIVTAC

Recibido: 19/julio/2020

Aceptado: 9/agosto/2020

Publicado: 25/septiembre/2020

${ }^{1}$ Argentina

\section{IIIInstitución}

Instituto Superior de Formación Docente

\section{Correo Eletrónico}

1marcelazeballos3@gmail.com

\section{iDORCID}

'https://orcid.org/0000-0002-8613-5998

\section{Citar así: GCAPA / IEEE}

Zeballos, M. (2020). Acompañamiento Pedagógico Digital para Docentes. Revista Tecnológica-Educativa Docentes 2.0, 9(2), 192-203.

https://doi.org/10.37843/rted.v9i2.164

M. Zeballos, "Acompañamiento Pedagógico Digital para Docentes", RTED, vol. 9, n. ${ }^{\circ}$ 2, pp. 192-203. sep. 2020.

\section{Resumen}

Una de las exigencias asignadas a los docentes es el cambio de sus prácticas por ser una necesidad de la era digital. Por tal motivo, es fundamental tomar distancia respecto de la práctica educativa, analizarla en cada una de sus dimensiones, por lo tanto, de esta manera descubrir su significado para poder realizar interpretaciones desde distintos ángulos. El proyecto tuvo como objetivo repensar las prácticas docentes mediadas por las Tecnologías del Aprendizaje y del Conocimiento (TAC) a partir de la implementación del programa de acompañamiento a docentes en la era digital. El desarrollo profesional guiado a través de actividades de seguimiento incluyó el trabajo colaborativo entre tutores y colegas con el objetivo de mejorar su práctica. El modelo de facilitación tuvo como centro del aprendizaje, a los docentes considerándolos como profesionales autónomos con capacidad para modificar su práctica. Se utilizó el enfoque cualitativo para realizar un análisis inductivo de los diferentes datos recolectados. Los resultados arrojados a partir de distintas estrategias de recolección de datos evidenciaron creencias, experiencias, resistencias, saberes, fortalezas y debilidades que los docentes poseían al integrar las tecnologías educativas en el aula. Con respecto a las conclusiones arrojadas se puede expresar que a pesar de los beneficios que trae la implementación de las tecnologías en la educación algunos docentes temen por llegada de nuevas herramientas digitales pues, consideran que puedan desplazarlos de su labor. La actitud de los docentes frente a la integración de las tecnologías educativas supuso un desafío en cuanto al cambio de paradigma en su cultura profesional.

Palabras clave: Acompañamiento,, prácticas docentes, tecnología, aprendizaje autoderminado.

\section{Abstract}

One of the demands assigned to teachers is to change their practices as it is a necessity of the digital age. For this reason, it is essential to distance yourself from educational practice, analyze it in each of its dimensions, therefore, in this way discover its meaning in order to make interpretations from different angles. The project aimed to rethink the teaching practices mediated by Learning and Knowledge Technologies (TAC) from the implementation of the teacher support program in the digital age. Guided professional development through follow-up activities included collaborative work between tutors and colleagues with the goal of improving their practice. The facilitation model had as a learning center, teachers considered as autonomous professionals with the ability to modify their practice. The qualitative approach was used to perform an inductive analysis of the different data collected. The results obtained from different data collection strategies showed beliefs, experiences, resistance, knowledge, strengths, and weaknesses that teachers possessed when integrating educational technologies in the classroom. Regarding the conclusions drawn, it can be expressed that despite the benefits that the implementation of technologies in education brings, some teachers fear the arrival of new digital tools because they consider that they can displace them from their work. The attitude of teachers towards the integration of educational technologies posed a challenge regarding the paradigm shift in their professional culture.

Keywords: Accompaniment, teaching practices, technology, self-determined learning. 


\section{Introducción}

Hargreaves (1996) afirmaba que la preparación de los profesores necesitaba ser repensada a la luz de los nuevos requerimientos de la sociedad de la información y del conocimiento. En este sentido, la profesión docente debería cambiar su rol para convertirse en creadores, gestores y curadores del conocimiento. Por lo tanto, el profesor tiene oportunidad de recrear, transformar su práctica, dar un nuevo significado a su trabajo para vivenciar una mayor satisfacción en su desempeño.

Para Santos-Guerra (1993) son múltiples los retos y desafíos que enfrentan los docentes del siglo XXI para atraer el interés de los estudiantes nacidos en la sociedad del conocimiento y del aprendizaje. En este sentido, los docentes deberían tender hacia un cambio de actitud sobre el mundo digital y de esta manera convertirse en creadores y gestores del conocimiento. Por lo tanto, para adoptar este nuevo rol docente será necesario la adquisición de competencias en el uso pedagógico de las tecnologías.

A pesar de los señalado, muchos docentes se resisten a integrar las tecnologías educativas como parte de su tarea de enseñanza. Es importante señalar, que los procesos de cambio e innovación a veces provocan en los docentes miedo, ansiedad o incertidumbre, para poder mejorar esto, será necesario un acompañamiento personalizado para crear una comunidad de aprendizaje basada en experiencias, creencias, emociones, colaboración incidiendo en la puesta en práctica de sus acciones.

Muchas veces los currículos, el espacio del aula, culturas institucionales, diseños de evaluación prescriptos frenan el cambio del docente y los anima a conservar métodos tradicionales y poco flexibles. Desde la perspectiva del cambio educativo, el acompañamiento al docente es una ayuda y apoyo para fomentar la consideración de las tecnologías en el proceso de enseñanza.

El objetivo del trabajo fue presentar la puesta en práctica de un proyecto de acompañamiento pedagógico a docentes para llevar a cabo la integración de las tecnologías del aprendizaje y del conocimiento. Las preguntas motivadoras de esta propuesta fueron las siguientes: ¿cómo cambian las personas?, ¿qué factores facilitan y obstaculizan el cambio?, ¿el cambio es metodológico o cultural?, ¿cómo integrar las tecnologías en el aula?, ¿cómo generar innovaciones en la era digital?, ¿cómo aprende el docente a través de las tecnologías digitales?

\section{Fundamentos del Programa}

Si pensamos en el docente como innovador de su práctica es fundamental considerar la dimensión personal o biográfica. Las historias vividas por el docente desde su formación inicial dejarán huellas, manifestándose en su desempeño profesional.

El autor Fullan (2013) alude que los grandes ejes de las reformas educativas deberían estar comandadas por principios pedagógicos. En este sentido es importante pensar en la relación entre tecnologías educativas y su uso pedagógico. Por lo tanto, en el contexto actual es necesario una formación en competencias digitales críticas para el uso seguro, comunicación, colaboración y creación del conocimiento.

Cuando se intenta integrar tecnologías en el aula, algunas investigaciones hacen referencia a las siguientes barreras:

1. Recursos.

2. Conocimiento.

3. Habilidades

4. Institución.

5. Actitudes.

6. Creencias.

7. Cultura relativa al contenido a enseñar.

Es importante que los docentes sean conscientes y reflexionen sobre estas barreras, ya que estarán mejor preparados y más atentos a encontrar soluciones. Antes de integrar las tecnologías al aula es fundamental realizar las siguientes preguntas: ¿qué queremos enseñar?, ¿cómo queremos enseñar? ¿qué características posee el sujeto pedagógico ¿en qué contexto vamos a enseñar? Por lo tanto, la integración pedagógica de las tecnologías se debería llevar a cabo dentro de un contexto. En síntesis, los docentes necesitan desaprender para reaprender y vincular todo esto con sus creencias, emociones, retroalimentaciones y de esta manera lograr un aprendizaje transformacional adquiriendo nuevos saberes, modificando sus actuaciones a luz de una reflexión crítica de sus marcos referenciales.

El acompañamiento al docente constituye un proceso continuo y planificado que permite recolectar información relevante para la toma de 
decisiones. En este sentido, una de las respuestas necesarias a los interrogantes y temas planteados está dado por el acompañamiento pedagógico, para mejorar el desempeño en el aula, con el propósito de responder a los requerimientos de la era digital.

\section{Aprender, Desaprender, Reaprender: en Búsqueda de un Aprendizaje Transformativo}

Damasio (2000) expresa la idea de considerar a los sentimientos como una parte del aprendizaje transformativo. Este enfoque se caracteriza por la posibilidad de generar un cambio a partir de explicitación de creencias, saberes y actitudes para internalizar nuevas perspectivas a partir de la reflexión. Esta descripción da cuenta que los sentimientos involucran ciertos procesos cognitivos, la persona realiza una elaboración mental de la experiencia vivida y la relaciona con vivencias previas que han sido significativas. Si bien el docente aprende a partir de la experiencia, Dewey, (1972) señala que no toda experiencia es aprendizaje. Para que esa experiencia se transforme en aprendizaje es necesario reflexionar críticamente.

Desde esta perspectiva el aprendizaje transformativo desafía a las personas a reflexionar críticamente sobre experiencias pasadas lo que conduce a un cambio en los sentimientos, creencias y actitudes.

Evidentemente, la actitud es un elemento fundamental para aprender. Las personas que son buenos aprendices son aquellas que supervisan su propio proceso de aprendizaje es decir someten la acción a la reflexión. Para los autores Marcelo \& Vaillant, (2009), el desarrollo profesional es asumido como espacio que nutre tanto la construcción de la personalidad como la identidad docente, entendiendo que el desarrollo profesional es el proceso a través del cual los docentes, en forma individual o colegiada, revisan, renuevan $y$ desarrollan su compromiso como agentes de cambio impulsados por propósitos morales.

\section{Aprendizaje Autorregulado}

Zimmerman (1986) señala que las personas tienen habilidades para estructurar una secuencia de pasos y decisiones que permitirán evaluar nuestras acciones para alcanzar metas. Los docentes que se autorregulan son promotores de su propio aprendizaje, esto se logra a través de la puesta en práctica de una serie de estrategias cognitivas, metacognitivas, motivacionales y conductuales.

El aprendizaje regulado permite activar y mantener la conducta, cogniciones y afectos en función de la tarea. Lograr esto es muy importante porque las personas que se autorregulan conocen mejor sus metas de aprendizaje, saben pedir ayuda cuando lo necesitan, monitorean su progreso y plantean nuevos desafíos. Por lo tanto, el proceso de autorregulación consiste en un espacio oportuno para practicar, de modo autónomo, habilidades y estrategias adquiridas previamente.

A partir de este proceso se reflexiona sobre el proceso de aprendizaje, se evalúa el rendimiento obtenido, así como la efectividad de estrategias utilizadas. El proceso finaliza cuando la persona es capaz de modificar la perspectiva utilizada, la realización de ajustes, para luego crear un ambiente más adecuado para su aprendizaje.

\section{Creencias, Emociones de los Docentes Frente a las Tecnologías del Aprendizaje y el Conocimiento}

Diversas investigaciones desarrolladas por Zaho (2002) puso en evidencia que las creencias de los docentes influyen en la utilización de las tecnologías en el aula. En el aula de clases, se observa que toda creencia influye en el aprendizaje, al mismo tiempo en sus innovaciones y en los proyectos de cambio a implementar. En síntesis, esas normas de comportamientos son creadas a partir de la relación con su entorno.

Gigliotti (2020) sostiene que la neurociencia sabe que al cerebro le gusta solamente dos cosas: Sobrevivir y Tener razón No le gustan los cambios porque eso le insume energía. Tenemos que lograr inculcar a los padres, docentes y a toda la gente involucrada en la educación, que los cambios cerebrales (influyendo fundamentalmente en las emociones y conductas) son semillas extraordinariamente valiosas para mejorar el rendimiento ético y social de las personas (p.2).

La enseñanza es una profesión emocional pues se establecen relaciones entre estudiantes y profesores. En una gran variedad de investigaciones se han abordado el tema creencias y emociones de los profesores en relación con sus prácticas pedagógicas.

En este sentido Ertmer (2005) ha identificado 
barreras para poder integrar las tecnologías, tales como creencias, emociones, falta de tiempo, y soporte técnico.

Aunque el camino es complejo a pesar de los obstáculos, las tecnologías también proporcionan oportunidades para la creación de conocimiento y para aprender colaborativamente. En este sentido, Montes \& Ochoa (2006), en un estudio de tipo exploratorio utilizando el método cualitativo, llevado a cabo con cinco profesores de una universidad privada, entre sus hallazgos encontraron que el uso de la tecnología tenía tres propósitos:

1. Para transmitir documentos, para comunicarse con los estudiantes (nivel básico).

2. Como herramienta para propiciar construcción de conocimiento (nivel medio).

3. Para generar comunidades de aprendizaje transformando las prácticas educativas mediadas por Tecnologías de la Información y la Comunicación (TIC) (nivel alto) y a toda la gente involucrada en la "educación", que los cambios cerebrales (influyendo fundamentalmente en las emociones y la conducta) son semillas extraordinariamente valiosas para mejorar el rendimiento ético y social de las personas.

Es relevante destacar, que el pensamiento centrado en la reflexión es el principal motor del docente, la llave que activa, la integración de las tecnologías en la institución educativa. Por otra parte, Carr (1996), al referirse a la educación expresa que es una actividad intencional desarrollada de forma consciente que sólo puede comprenderse en relación con el marco de pensamiento en cuyos términos dan sentido a los practicantes a lo que hacen y a lo que tratan de conseguir. En ese sentido, retomando los planteamientos de Contreras (1987), en la intencionalidad del maestro, se pueden distinguir tres miradas diferentes: el experto técnico, el reflexivo e intelectual crítico.

\section{La Integración de Tecnologías Educativas en el Aula}

La integración de las tecnologías educativas es tratada por Torres (2012), quien plantea cuatro niveles de integración que vinculan el conocimiento y uso de las TIC por parte de los docentes: nivel de preintegración, nivel de integración básica, nivel de integración media, nivel de integración avanzada que se evidencian en los niveles manejados por otros autores (Jaramillo, Castañeda \& Pimienta, 2009).

En este proceso de integración de las tecnologías educativas se puede señalar que el nivel de preintegración se refiere a un primer acercamiento en el uso de las TIC donde el docente aplica teorías constructivistas en el proceso de aprendizaje, realiza prácticas escolares, maneja herramientas comunicativas, aplica el desarrollo de bases de datos en ejercicios con los estudiantes. Los niveles de integración del tic permiten al docente evaluar en cuál de ellos está y a cuál puede avanzar. De acuerdo con lo mencionado, se presenta los siguientes niveles de integración de las tecnologías educativas:

1. El nivel de integración el docente realiza búsquedas de información para su clase, utiliza medios mixtos tanto audiovisuales como computacionales, maneja editores de imágenes, videos, utiliza la web para buscar información relacionada con sus temas de clase.

2. El nivel de integración media está centrado en acciones del docente relacionadas con la utilización de sitios web para ver aplicaciones educativas, realiza páginas de edición de contenidos con sus recursos personales, utiliza recursos tecnológicos reemplazando los manuales, elabora cursos virtuales, interactúa con sus estudiantes a través de las redes sociales.

3. El nivel de integración avanzada se caracteriza por un trabajo más especializado del docente se evidencia en: diseño de ambientes de aprendizaje, maneja estrategias para el uso de TIC en educación.

Al respecto, Lozano (2011), señala, que hay que hablar menos de TIC y más de las Tecnologías del Aprendizaje y del Conocimiento (TAC) ya que nos ayudarán a:

1. Promover la autonomía de los estudiantes.

2. Despertar la curiosidad. 
3. Seleccionar, localizar y evaluar la información.

2. Promover una educación más personalizada.

3. Indagar las ventajas que tienen las redes, recursos y herramientas.

4. Descubrir posibilidades pedagógicas de los recursos digitales y también cuáles son sus potencialidades comunicativas.

5. Promover el uso responsable en el uso y el aprovechamiento de la información de una manera ética.

Las estrategias tecnológicas evolucionan hacia espacios más formativos promoviendo el aprendizaje colaborativo y la reflexión crítica del uso de las tecnologías. En este sentido, Imbernon (2011), señala la importancia de experimentar en el terreno de habilidades, capacidades, actitudes, al mismo tiempo cuestionarse los valores y concepciones de cada docente. La reflexión como herramienta fundamental para el aprendizaje debería ser considerada como un espacio habitual en el cual podemos mejorar el desarrollo profesional docente. Algunas estrategias que se pueden implementar. son reflexiones narrativas, resolución de incidentes críticos, investigación de diferentes temáticas, resolución de problemas.

Perea (2014) hace énfasis en el hecho que la investigación muestra la importancia en la formación de los docentes, la infraestructura que deben tener las aulas y el nivel de manejo de herramientas que el profesor debe adquirir para la sociedad de estos tiempos. Las tecnologías han llegado a las vidas de las personas, su estudio, entrenamiento y su forma de entender el mundo. Desde esta perspectiva, Mujica (2020) sostiene que la tecnología cuando es utilizada de manera adecuada permite que los estudiantes logren ser autosuficientes y adquieran habilidades que mejoren su desempeño profesional. El uso pedagógico de las tecnologías permite que los alumnos sean más activos, responsables y con un pensamiento más flexible.

\section{Desarrollo}

Las tecnologías han introducido cambios en la educación, pasando de un aprendizaje basado en el docente, hacia un paradigma basado en el estudiante.

En este sentido, el profesor debe adquirir nuevas competencias que le permita enfrentar los retos de la sociedad del conocimiento. Para ello, debe apropiarse de las tecnologías educativas para mejorar su labor y el aprendizaje en el aula.

En consecuencia, para lograr dichas competencias digitales será necesario un programa acompañamiento a docentes orientado a la mejora profesional. El desarrollo profesional situado ayuda a identificar las situaciones que inciden en los aprendizajes y desarrollar competencias en los docentes para el inicio del camino de la mejora escolar. En este sentido, todo programa de acompañamiento debe generar las condiciones propicias facilitando la reflexión crítica como instrumento esencial del cambio educativo.

Uno de los objetivos centrales de todo proceso de acompañamiento es la construcción compartida entre personas en un determinado contexto. Por tal motivo, es fundamental destacar el rol del tutor como facilitador de aprendizajes en entornos digitales. En definitiva, podemos decir que es un rol fundamental para propiciar climas y ambientes de aprendizajes adecuados para el desarrollo efectivo de aprendizajes significativos. ¿Por qué acompañamos a los docentes pedagógicamente en la era digital?

1. Mejoró la práctica pedagógica.

2. Aumentó la motivación y autonomía de los docentes.

3. Favoreció el aprendizaje autodirigido.

4. Enfatizó el uso pedagógico de las tecnologías.

¿Para qué acompañamos?

1. Problematizar el uso pedagógico de las tecnologías educativas.

2. Fortalecer el trabajo en equipo.

3. Explicitar creencias y actitudes con respecto a las tecnologías educativas.

4. Reflexionar sobre cómo se aprende en la era digital.

Mediante estas reflexiones los docentes expusieron:

1. Distintos modelos didácticos utilizados en su práctica.

2. Actitudes frente a las tecnologías educativas.

3. Barreras o dificultades para integrar las tecnologías a su práctica docente. 
La importancia del programa radica fue acompañar a los docentes a iniciar un proceso desde la introspección a la reflexión de tal forma que cada uno de los docentes tome conciencia por el proceso por el que transita. En el presente programa se utilizó el modelo TPACK como enfoque para planificar con tecnología. El programa didáctico no se centró en la herramienta sino en los estudiantes, así como en el currículo. Toda planificación que realizaron los docentes fue desde una concepción situada y adaptada al contexto y basada en actividades. No obstante, es importante, reflexionar sobre el siguiente interrogante; ¿Qué necesita saber un profesor para planificar y desarrollar su tarea como docente?

1. Conocimiento disciplinar.

2. Conocimiento pedagógico (general especifico)

3. Conocimiento sobre tecnología

4. Conocimiento tecnológico disciplinar.

5. Conocimiento tecnológico pedagógico.

6. Conocimiento tecnológico pedagógico disciplinar.

El docente trabajó con su disciplina, con sus conocimientos pedagógicos, tecnológicos, didácticos y a su situación contextual. El conocimiento utilizado en el contexto escolar requirió ser producido de manera colaborativa, pues más que trabajar sobre los propios saberes, se estableció la relación con los conocimientos de los colegas para el logro de un proyecto común. Este conocimiento incluyó:

1. Conocimiento del distrito o comunidad en donde trabaja.

2. Conocimiento de la cultura escolar

3. Conocimiento de los estudiantes

\section{Descripción del Proyecto}

Todo proceso de acompañamiento se inició con una etapa de diagnóstico de necesidades llevando implícita la detección de necesidades formativas de los destinatarios.

Los objetivos del programa fueron los siguientes:

5. Reflexionar sobre las creencias que sustentan la práctica.

6. Evaluar los entornos personales de aprendizaje.
1. Potenciar el uso pedagógico de las tecnologías educativas e integrarlas como una herramienta más en el proceso de enseñanza-aprendizaje en el nivel superior.

2. Favorecer la innovación en el contexto de las prácticas reales de los profesores.

3. Desarrollar en los docentes, competencias básicas necesarias en el uso y manejo de las TIC, TAC y Tecnología del Empoderamiento y la Participación (TEP) para enriquecimiento de sus prácticas pedagógicas.

4. Enseñar a utilizar recursos personales para conocer, desarrollar y gestionar las emociones, de tal modo, favorecer el bienestar personal en contextos digitales.

\section{Análisis del Contexto}

El contexto da forma a todo programa que tenga como objetivo acompañar a los docentes. Evaluar el contexto permite pensar que el aprendizaje no ocurre en el vacío. Todo proyecto educativo tiene que articularse con el entorno. Los factores contextuales pueden facilitar o ser un obstáculo para el desempeño docente. Por tal motivo, el primer paso que se tuvo en cuenta para el diseño del programa fue identificar y examinar los siguientes componentes: condiciones institucionales, condiciones áulicas y políticas educativas.

\section{Característica del Destinatario}

El programa fue diseñado para docentes del Nivel Primario, Inicial y Formación Docente. La antigüedad de los profesores osciló entre 3 a 10 años. Algunos participantes se desempeñaban en establecimientos estatales y otros en instituciones de gestión privada. Los docentes del Nivel Superior impartían disciplinas como Didáctica y Pedagogía.

\section{Docentes Mentores o Acompañantes}

El mentor colega adoptó una postura de igualdad frente a los profesores posibilitando el intercambio ideas para reflexionar sobre ellas. Compartió sus conocimientos con los profesores, mostrando ejemplos y situaciones problema. La función del mentor dio lugar a un liderazgo situacional en estrecha relación con factores tales como, el clima, los vínculos, la gestión de emociones, 
la autorregulación de los aprendizajes y factores contextuales que inciden en el aprendizaje.

Entre las funciones del profesor mentor se destacaron:

1. Ser capaces de ayudar a develar creencias, presupuestos, valores subyacentes en la práctica de los profesores.

2. Ayudar a los profesores a encontrar o identificar nuevos enfoques didácticos $\mathrm{y}$ tecnológicos

3. Conocer como a través de la metodología de investigación se puede reflexionar sobre la práctica docente.

Con respecto a los roles del profesor mentor podemos mencionar:

1. Construcción de una relación de confianza a partir de vínculos con los docentes comprometiéndose con su desarrollo personal y profesional.

2. Generación de puentes o andamiajes para lograr construir conocimiento.

3. Moderar espacios de comunicación.

4. Llevar seguimiento de los alumnos.

5. Realizar tutorías online.

Los mentores llevaron a cabo el programa de acompañamiento a través de un enforque interdisciplinar.

1. Mentor pedagogo.

2. Mentor con especialidad en tecnología.

3. Mentor neurocientífico.

\section{El Trabajo por Competencias}

Se implementó un enfoque integrado de competencias apuntando a procesos metacognitivos utilizados para evaluar capacidades y conocer el estilo de aprendizaje de los estudiantes. En definitiva, la competencia del educador en estos contextos cambiantes requirió no solo del saber sino del saber hacer y del saber ser.

Competencias desarrolladas:

\section{Competencia socioemocional:} Capacidad para tomar conciencia en gestión de las emociones.
1. Competencia

pedagógica:

Desarrollo de una clara conciencia de sus capacidades personales, confianza en sí mismos generando entornos de aprendizajes colaborativos. Capacidad para utilizar Tecnologías de la Información y la Comunicación.

2. Competencia cognitiva: Dominio de estrategias metacognitivas y estrategias cognitivas. Capacidad para aprender con otros en redes personales de aprendizaje

3. Competencia contextual: Adaptación de sus conocimientos disciplinares, pedagógicos, tecnológicos, didácticos a situaciones contextuales.

4. Competencia digital: Desarrollo del pensamiento crítico frente a las tecnologías educativas.

\section{Metodología}

El énfasis estuvo puesto en los factores contextuales, la institución educativa en donde surgen la mayor parte de las problemáticas. La mayoría de las veces el docente no poseía tiempo para reflexionar sobre sus acciones profesionales. El enfoque didáctico que se utilizó fue el aprendizaje por exploración y descubrimiento en la web. Las propuestas metodológicas utilizadas fueron: porfolios electrónicos, Wesquets y clase invertida.

Se ofreció oportunidades de prácticas guiadas en línea con la finalidad de desarrollar grados crecientes de autonomía, siendo el estudiante un protagonista activo y el docente un mediador de situaciones de aprendizaje. Para desarrollar las distintas competencias se planificaron actividades en las cuales variaron en el grado de complejidad. Las actividades se caracterizaron por ser de autenticidad alta a partir de situaciones reales. Se utilizaron distintas herramientas digitales como grupos de Facebook, WhatsApp, Blog, Google Classroom, Google Drive y muros colaborativos.

Para evitar deserción del curso online se tuvieron en cuenta las siguientes actividades:

1. Espacio para la inducción: Familiarización con la plataforma. 
2. Trayecto de aprendizaje: Se dio a conocer el trayecto que realizó el cursante para alcanzar los objetivos y competencias del curso.

3. Característica de los estudiantes: Se facilitó diferentes recursos adaptados a los distintos estilos de aprendizaje.

4. Aspecto comunicativo: Se fundó confianza de manera permanente para la comunicación y retroalimentación.

5. Seguimiento: Se generó informes periódicos sobre la participación de los estudiantes.

6. Socialización en línea: Se creó espacios para el intercambio de expectativas, necesidades, esperanzas, ansiedades y gestión del tiempo.

\section{Estrategias didácticas}

Las estrategias cognitivas se organizaron desde un procesamiento más superficial a uno que requirió un pensamiento de mayor aprendizaje. Algunos ejemplos fueron repasar, elaborar, organizar y pensar de manera crítica. En la medida que seleccionamos, organizamos y reflexionamos críticamente sobre la información esto se fue convirtiendo en una manera de estudiar por parte de los docentes. El uso y la organización del tiempo fue una de las variables que incidió en el proceso de aprendizaje.

Se utilizó Moodle como plataforma virtual, su flexibilidad, derivada de su estructura modular, garantizó el soporte a los distintos estilos de aprendizaje. Un Entorno Virtual de aprendizaje, es una aplicación informática diseñada para facilitar la comunicación pedagógica entre los participantes en un proceso educativo, sea éste virtual, bimodal o presencial con apoyo de tecnologías (Adell, Castellet \& Gumbau, 2004).

Durante el proceso se pudo observar como la herramienta tiene poca utilización en los procesos formativos. En algunos casos los profesores nos informaron que habían utilizado la herramienta por iniciativa propia para subir información e intervenir en los foros de discusión.

Para crear un ambiente de aprendizaje motivador fue necesario mantener el curso organizado y motivante para los estudiantes, actividades promotoras de participación, retroalimentación asertiva y construcción de espacios basados en el vínculo.

\section{Recursos Digitales}

Un recurso digital es cualquier elemento que esté en formato digital, se puede visualizar y almacenar en un dispositivo electrónico. La idea principal fue que el uso de los recursos estuvo al servicio de la planificación docente y a su vez se tuvo una clara intención educativa. Lo fundamental fue monitorear constantemente el diseño y la selección de los recursos para asegurarnos que los estudiantes pudieran acceder. Algunos recursos que se tuvieron en cuenta fueron videoconferencias, presentaciones, podcast, multimedia, chat, foros $\mathrm{y}$ pizarras electrónicas.

\section{Evaluación de los Aprendizajes}

La evaluación diagnóstica durante mucho tiempo era aquella que se realizaba al principio para recolectar los saberes previos de los estudiantes. Con el transcurso de nuevos paradigmas la evaluación inicial se llama hoy evaluación diagnóstica continua porque estamos diagnosticando constantemente a los estudiantes. En este sentido, este tipo de evaluación busca dos objetivos: primero, entender en qué estado se encuentran los estudiantes a principio del ciclo lectivo, y segundo, tomar decisiones que faciliten la toma de daciones para la mejora de los aprendizajes.

Durante la aplicación del programa la retroalimentación fue específica, favoreció la oportunidad para recolectar evidencia sobre el aprendizaje de los cursantes con finalidad de mejorar el entorno de aprendizaje. Existen dos tipos de retroalimentación: focalizadas en las emociones y focalizadas en la tarea. Es importante señalar que a partir de una buena retroalimentación emocional se consigue que las evaluaciones sean bien recibidas por parte de los estudiantes. En relación con este punto, se considera también que la retroalimentación es fundamental para el estudiante porque le permite entender, sus errores y cómo corregirlos.

El error formó parte del proceso de aprendizaje dentro de un clima tranquilo y con oportunidades de interacción. Se hicieron explícitos los criterios de evaluación para que los docentes supiesen lo que se esperaban de ellos. Estos criterios fueron públicos y compartidos. A partir de esta estrategia, los docentes tuvieron la oportunidad de revisar sus trabajos y generaron otras estructuras de pensamiento para la construcción de conocimientos. 
La retroalimentación proporcionó a los docentes una explicación de sus fortalezas y debilidades. Se utilizó la técnica de feedback ofreciendo información sobre futuras mejoras, la comunicación fue asertiva y respetuosa. La evaluación formativa fue fundamental para que los estudiantes regularan su propio proceso de aprendizaje, reconocieran sus fortalezas, debilidades y monitorearan las estrategias utilizadas en sus aprendizajes.

La evaluación sumativa tuvo la función de articular, relacionar e integrar todos los saberes aprendidos a lo largo del proyecto. Fue importante establecer relaciones y vinculaciones de todo el proceso de aprendizaje. Una de las herramientas de evaluación sumativa más relevante fue la carta personalizada. La retroalimentación estuvo centrada en la persona y en su autorregulación. Los mensajes fueron claros y con sugerencias de mejora.

Los criterios utilizados para redactar la carta se basaron en: respetar los estilos de aprendizaje de los docentes, tener en cuenta las diversas evidencias observadas en foros, blog y en los porfolios del docente. Para obtener evidencia sobre el aprendizaje recolectamos información a partir de distintas estrategias:

1. Utilización del blog como estrategias didácticas para la expresión de creencias, vivencias y sentimientos.

2. Diarios reflexivos: estrategia didáctica que permitió desarrollar habilidades metacognitivas. Consistió en la reflexión y la descripción del proceso de aprendizaje.

3. Rúbricas: herramienta de evaluación que ayudó al docente a pensar en profundidad y a autoevaluarse.

Los objetivos de la evaluación apuntaron a reconocer el uso pedagógico de las tecnologías educativas al momento de planificar sus actividades de clase e identificar los cambios de creencias, actitudes frente a las tecnologías educativas. Teniendo en cuenta los objetivos planteados, los instrumentos de evaluación sirvieron para mejorar la calidad de los aprendizajes y el desempeño de los docentes. Dentro de los instrumentos de evaluación utilizados fueron muy útiles las rúbricas, el cuestionario online, el blog y el cuaderno de narraciones.

\section{Resultados}

\section{Evaluación del Programa}

Otro aspecto destacable fue la calidad técnica del programa. Dentro de este criterio abordamos la evaluación para determinar en qué medida se atiende a la diversidad y estilos de aprendizaje. Se ofreció una gran cantidad de actividades individuales y grupales las cuales desarrollaron distintas habilidades de pensamiento, crear, evaluar, analizar, comprender, aplicar y evaluar. En este punto debemos expresar las variedades de actividades que apuntaron a observar videos, participar en debates, tener una conversación, realizar búsquedas de selección, evaluación de la información y construir conocimiento.

La adecuación al contexto en relación con las necesidades de los docentes fue muy positiva. Durante todo el programa se planteó un sistema de identificación de las necesidades de formación. Las actividades llevadas a cabo aluden al interés y motivación evidenciados por los docentes. Las actividades que más despertaron interés han sido los trabajos colaborativos, la adquisición de herramientas para la gestión de información, curaduría de contenidos, estrategias para desarrollar video conferencias, y herramientas tecnológicas para evaluar los aprendizajes.

El vínculo entre los tutores y los estudiantes fue fundamental para que los estudiantes se sientan incentivados $y$ de esta manera mejoraran el rendimiento académico. Durante todo el proceso prevaleció la comunicación fluida, dentro de un clima cálido y con un acompañamiento constante de las actividades que realizaban los participantes. Por todo esto, fue necesario definir los criterios a tener en cuenta durante la comunicación para favorecer la calidez entre los participantes.

Los resultados fueron analizados desde un enfoque de triangulación para garantizar confiabilidad al estudio. El diario de campo y la reflexión crítica como estrategias para mejorar la reflexibilidad fueron necesarias para otorgar validez a los datos. Se utilizaron distintas herramientas de recolección de datos: grupos de discusión, narraciones de los docentes y tutores, análisis de documentos y producciones de los participantes. Fue interesante como los docentes iban reflexionando sobre su desarrollo profesional y su relación con las tecnologías educativas a través de sus expresiones. 
Un elemento que apareció reiteradamente fue el temor y las limitaciones con respecto al uso pedagógico y didáctico de las tecnologías educativas. Sin duda, la integración de las tecnologías en el aula puede ser complicado por varios aspectos, se puede tener miedo a perder el control del aula, a poseer menos conocimientos que los estudiantes y el miedo a equivocarse.

Los docentes no poseen los conocimientos, habilidades y actitudes necesarias para utilizar con fines pedagógico las tecnologías educativas. Existen resistencias personales al cambio pedagógico que representa la cultura digital. Muchos se sienten incómodos, incapaces y con temor en el uso de herramientas tecnológicas. Es importante señalar, que la resistencia al cambio no se atribuye sólo al sujeto, existen condiciones institucionales que a veces obstaculizan la posibilidad del cambio.

Muchas experiencias expresadas por los docentes utilizando las tecnologías se basaron en tareas individuales y esporádicas. Durante las diferentes narraciones se reconoce a las tecnologías como herramientas que enriquecen el aprendizaje. Unos de los aspectos que apareció reiteradamente fue la escasez de herramientas tecnológicas en las instituciones educativas. Los docentes expresan: "Los materiales tecnológicos llegaron y los docentes no saben usarlo. Yo enchufé y comencé a tocar todo sin el permiso de nadie". "Los técnicos llegan tarde, le dije a los niños que exploren. En la escuela las herramientas tecnológicas están guardados porque están esperando que alguien nos enseñe a usarlas".

Con respecto al trabajo colaborativo de los docentes se observó que el individualismo es la cultura dominante de los profesores. Uno de los factores fue la soledad y la ausencia de acompañamiento cuando imparten sus clases con tecnología. Otro factor que apareció reiteradamente fue la escasa comunicación con sus colegas y con muy poco tiempo para diseñar planificaciones en colaboración con sus pares.

La utilización de la herramienta de videoconferencia Zoom fue muy efectiva para la organización de grupos colaborativos. Durante las distintas sesiones se observó que los docentes iban flexibilizando su esquema tradicional de aprender. A la pregunta ¿qué aprendieron a partir del programa y qué necesitan aprender? Surgieron conversaciones muy interesantes ya que facilitó discusiones sobre sus problemas y limitaciones para integrar las tecnologías a la clase.

La utilización de Facebook como herramienta colaborativa favoreció el trabajo entre pares a partir de:

1. Compartir documentos, infografías, artículos y videos relacionados con la integración de las tecnologías educativas en el aula.

2. Documentar el trabajo colaborativo utilizando herramientas complementarias, tales como murales colaborativos, documentos colaborativos y blog.

3. Organización de la herramienta como banco de recursos digitales.

4. Expresión de creencias, saberes y experiencias relacionadas con la integración de las tecnologías educativas.

Durante los distintos debates en el foro de discusión una docente expresa: "Muchas veces vemos a la tecnología como herramienta técnica y con un uso poco reflexivo". A partir de esta expresión se trabajó sobre las creencias de confundir a las computadoras con un mejor aprendizaje y con la innovación educativa. El docente posee creencias fundamentadas desde su paso por la formación docente. Cuando comienza a dar clase recibe un gran choque con el contexto escolar. Además, uno de los factores que influyó en las creencias de los docentes fue la cultura escolar la cuál incidió en el desarrollo profesional. La estrategia que se empleó para trabajar las creencias fue el desarrollo profesional a través de la investigación acción a partir de la cual el docente indagó su práctica de manera reflexiva. El objetivo de esta actividad fue visibilizar las creencias para alcanzar una comprensión critica de su práctica docente.

Una de las herramientas utilizada para dicha actividad fue Symbalaoo que permitió organizar enlaces y diferentes sitios. Se observó distintos recorridos en el desarrollo profesional docente, como angustia y miedo al desear integrar las tecnologías educativas a la clase. Por otro lado, había docentes preocupados por experimentar en nuevas metodologías didácticas y otros docentes, ante esta situación algunos docentes manifestaron resistencia al cambio.

\section{Conclusiones}

Como conclusión general del programa de 
acompañamiento, cabe señalar que el éxito del acompañamiento pedagógico depende de la actitud de los docentes dentro del proceso de aprendizaje. Se requiere apertura a la crítica, el interés por aprender, por desaprender y el análisis crítico del contexto. Por otra parte, los datos derivados de los distintos instrumentos de recolección de datos muestran que el docente es consciente de que las tecnologías educativas son herramientas que deben conocer y aplicar a la práctica educativa.

El aprendizaje autónomo es condición para continuar aprendiendo, pero es preciso señalar que no ha tenido un espacio propio en los currículos escolares. Pareciera ser que es un aprendizaje que se da por supuesto y, muchas veces se le reduce a un conjunto de técnicas de aprendizaje. Las estrategias utilizadas para la generación de aprendizaje autónomo plantearon a los docentes, las siguientes decisiones:

a. ¿Qué contenidos son relevantes para trabajar con los estudiantes?

b. ¿Qué actividades son las más adecuadas atendiendo a las necesidades de los estudiantes?

c. ¿Qué criterios utilizar a la hora de evaluar los recursos digitales?

d. ¿Todos los recursos digitales pueden ser utilizados como recursos pedagógicos?

Todo desarrollo profesional debería ser pensado dentro de las biografías, creencias y el lugar específico donde el docente desarrolla su actividad. Las prácticas profesionales se verán modificadas si se propician procesos de reflexión colaborativa.

Integrar las tecnologías no sólo depende de la calidad técnica y de sus posibilidades pedagógicas sino también de metodologías, creencias, acciones, emociones y actitudes de los profesores. Diferentes barreras dificultan la integración de las tecnologías por parte de los docentes, el tiempo, la capacitación, el currículo, apoyo para usar de la tecnología y el cambio en el rol.

El desaprendizaje y el reaprendizaje son procesos imprescindibles para facilitar la reflexión crítica de los esquemas de pensamiento. No se trata de destruir los viejos marcos teóricos sin aprender de ellos, sino de analizar qué aspectos recuperar de lo viejo para integrar parte de ello en la construcción de lo nuevo. Es importante señalar que el proceso no es solamente cognitivo, también es emocional.

Los elementos que se debería tener presente en este proceso de aprender y desaprender son los siguientes:

1. Cuestionamiento de actitudes y supuestos arraigados.

2. Creación de ambientes seguros para expresarse con libertad.

3. Desafío de actitudes ante viejos paradigmas.

4. Poner prueba actitudes, saberes y conductas a partir de la discusión con otros.

Hay una variedad de estrategias para lograr el aprendizaje transformativo siempre y cuando se cree un ambiente donde el pensamiento crítico y el cuestionamiento sea aceptado. Los estudiantes adultos tienen una gran variedad de experiencias previas que contribuyen al aprendizaje de los demás. Es fundamental que los tutores consideren estas experiencias y las activen. Por lo tanto, los adultos están listos para aprender cuando ven que lo que van a aprender ayudará a resolver problemas de su contexto.

Es importante señalar, que el reto de las instituciones educativas será innovar no sólo en tecnologías educativas, sino también en las concepciones y prácticas pedagógicas, lo que significa cambiar el modelo de enseñanza en su totalidad: cambios en el rol del docente, en las actividades de aprendizaje, en la forma de organizar el espacio escolar, recursos materiales, capacitación situada y los actores involucrados comprometidos en un proyecto educativo construido colaborativamente.

Para una verdadera integración de las tecnologías en las instituciones educativas se requiere de una visión holística que comprenda a las políticas educativas y a la organización escolar. Cabe señalar que, las políticas de integración de las tecnologías en educación tienen mayor éxito cuando se incluye capacitación desde el enfoque situado basada en competencias y actividades que integran a las tecnologías en la práctica de aula.

Por otra parte, la integración de las tecnologías educativas en la institución educativa, deben contar con una metodología institucionalizada y centrada en el uso didáctico y pedagógico de las tecnologías. Por tanto, para iniciar ese camino se debe comenzar con un análisis previo que arroje el nivel de apropiación de las tecnologías por parte de los docentes. 
En cuanto a los desafíos, el presente programa de acompañamiento a docentes en la era digital deberá continuar analizando y monitoreando su aplicación en distintos contextos educativos, con la intención de ajustar y transformar para tender a la mejora continua.

\section{Referencias}

Adell, J., Castellet, J. M., \& Gumbau, J. P. (2004). Selección de un entorno virtual de enseñanza y aprendizaje de código fuente abierto para la Universitat Jaume I. Castelló: Centre d'Educació i Noves Tecnologies (cent) de la Universitat Jaume I. Recuperado de: http://cent.uji.es/doc/eveauji_es.pdf.

Contreras Domingo, J. (1987). De estudiante a profesor. Socialización y aprendizaje en las prácticas de enseñanza. Revista de Educación, (282), 203-231.

Damasio, A. R. (2000). Sentir lo que sucede. Editorial Andrés Bello, Santiago de Chile. (Título orig.: The Feeling of What Happens: Body and Emotion in the Making of Consciousness. Harcourt, New York. 1999).

Dewey, J. (1972). El niño y el programa escolar. Losada.

Ertmer, P. (2005). Teacher Pedagogical Beliefs: the final frontier in our quest for technology integration. Educational technology, research and development, 53 (4), 25-40.

Fullan, M. (2011). Choosing the Wrong Drivers for Whole System Reform. Centre for Strategic Education, Seminar Series 204.

Gigliotti, J. J., Gigliotti, J. M., \& Treco, D. (2020). El arte de decidir, cómo equilibrar la razón con la emoción, ¿quién nos enseña? Revista Tecnológica-Educativa Docentes 2.0, 9(1), 3436. https://doi.org/10.37843/rted.v9i1.88

Hargreaves, A. (1996). Profesorado, cultura y posmodernidad. Cambian los tiempos, cambian los profesores. Morata

Inbernón, F. (2011). Un nuevo desarrollo profesional del profesorado para una nueva educación. Revista de Ciencias Humanas, 12 (19), 75-86.

Jaramillo, P., Castañeda, P. \& Pimienta, M. (2009). Qué hacer con la tecnología en el aula: inventario de uso de las TIC para aprender y enseñar. Educación y Educadores, 12 (2), 159-179.

Lozano, R (2011). Las 'TIC/TAC': de las tecnologías de la información y comunicación a las tecnologías del aprendizaje y del conocimiento. Recuperado de: http://www.thinkepi.net/lastic-tacde-las-tecnologias-de-la-informacion-y-comunicacion-alas-tecnologias-del-aprendizaje-y-del-conocimiento.

Marcelo García C. y Vaillant, D. (2009). Desarrollo profesional docente. Narcea.

Martinez, E y Sánchez, S (2002). La tecnología en las aulas. El profesor como orientador de los procesos de enseñanza-aprendizaje. Federación Andaluza de Centros de Estudios Privados.

Mujica-Sequera, R. (2020). E-Learning como estrategia pedagógica en la educación superior. Revista Tecnológica-Educativa Docentes 2.0, 9(1), 37-41. https://doi.org/10.37843/rted.v9i1.103
Montes, J. y Ochoa, S. (2006). Apropiación de las tecnologías de la información y la comunicación en cursos universitarios. Acta Colombiana de Psicología, 9 (2), 87-100.

Perea Aguayo, A (2014). Importancia de los recursos tecnológicos en el aula, formación de los docentes y manejo de herramientas tecnológicas. Recuperado de: http://tauja.ujaen.es/bitstream/10953.1/1244/1/TFG_PereaAguay o,Almudena.pdf.

Santos Guerra, M. (1993). La evaluación, un proceso de diálogo, comprensión y mejora. Archidona.

Torres-Ortiz, J. (2012). Incidencia de Moodle en las prácticas pedagógicas en modalidad educativa B-Learning. Revista de Investigación, Desarrollo e Innovación, 2 (2), 39-48. Recuperado de:

http://revistas.uptc.edu.co/index.php/investigacion_duitama/artic le/view/1315

Zhao, Y. (2002). Conditions for Classroom Technology Innovations. Teachers College Record, New York, v.104, n.3.

Zimmerman, B. J., \& Martinez-Pons, M. (1986). Development of a structured interview for assessing student use of self-regulated learning-strategies. American Educational Research Journal, 23(4), 614-628. 